\title{
Self-force approach to synchrotron radiation
}

\author{
Lior M. Burko ${ }^{\text {a) }}$ \\ Theoretical Astrophysics, California Institute of Technology, Pasadena, California 91125 \\ and Department of Physics, Technion-Israel Institute of Technology, 32000 Haifa, Israel
}

(Received 7 June 1999; accepted 16 September 1999)

\begin{abstract}
We analyze radiation reaction for synchrotron radiation by computing, via a multipole expansion, the near field and derive from it the Lorentz four-force, which we evaluate on the world-line of the charge. We find that the temporal component of the self four-force agrees with the radiated power, which one calculates in the radiation zone. This is the case for each mode in the multipole decomposition. We also find agreement with the Abraham-Lorentz-Dirac equation. (C) 2000 American Association of Physics Teachers.
\end{abstract}

\section{INTRODUCTION AND OVERVIEW}

Radiation reaction is a century-old problem of physics, which has been dealt with via many approaches during the years. (For reviews see, e.g., Refs. 1-3.) Much of the understanding we currently have on radiation reaction involves electromagnetic theory, ${ }^{4,5}$ but similar problems arise also in other branches of physics, e.g., in gravitation theory ${ }^{6}$ (and even in fields such as acoustics ${ }^{7}$ ).

The usual approach for radiation reaction is based on arguments relating to the balance of quantities, which are constants of motion in the absence of radiation reaction, specifically the energy and angular momentum. Such balance arguments involve integration of the flux of an otherwise conserved quantity over a boundary which normally consists of a distant sphere. This approach yields the energy and angular momentum radiated per orbit (i.e., the time-averaged energy and angular momentum fluxes). Although such methods have been quite useful for the analysis of many problems involving radiation reaction, they are unsatisfactory for three main reasons. First, they apply only to periodic systems. Systems which involve radiation reaction are in a strict sense not periodic, because of the loss of energy through radiation. To illustrate the next point, consider a system comprised of a compact object in motion around a black hole, which is radiating gravitational radiation. Even if the system is approximated by a quasi-periodic model, assuming that the radiation reaction effects are small (specifically, that the time scale for inspiraling is much larger than the orbital period), eventually the orbit evolution due to radiation reaction will be fast. Recall now that the usual approach for radiation reaction yields only the time-averaged energy and angular momentum fluxes. This leads us to the second problem with the usual approach: During the last stages of inspiral the evolution of the orbital motion is fast, such that the time-averaging methods would be inaccurate. The third problem occurs only for systems which in the absence of radiation reaction involve nonadditive constants of motion. The usual approach is based on a balance argument for conserved quantities. Specifically, knowledge of the radiated power at infinity implies that the system underwent a loss of energy at an equal rate because of total energy conservation. However, when nonadditive constants of motion are involved, one can no longer add up the conserved quantity in the system and that part of the quantity which escaped to infinity, and then use the conservation law to deduce from the latter on the former. Such nonadditive constants of motion occur for the very interest- ing case of motion in the space-time of a spinning black hole, which is likely to be observed by future gravitationalwave observatories.

An alternative approach is to calculate the radiation reaction directly, at the position of the system, rather than indirectly by balance arguments and calculation only of the far field in the radiation zone. The first such method was used by Dirac $^{4}$ for obtaining the Abraham-Lorentz-Dirac equation for an electric charge in arbitrary motion in Minkowski space-time. In that approach one imposes local conservation laws on a tube surrounding the world-line of the particle, and integrates the conservation law across the tube, thus obtaining the equations of motion, including the radiation reaction effects. However, as is frequently the case with problems involving radiation reaction, the force which acts on the charge diverges. Dirac removed this divergence by using the so-called radiative Green's function, instead of the retarded Green's function. Specifically, one can write the retarded Green's function $G^{\text {ret }}$ as the sum of two terms, namely,

$$
\mathrm{G}^{\mathrm{ret}}=\frac{1}{2}\left(\mathrm{G}^{\mathrm{ret}}+\mathrm{G}^{\mathrm{adv}}\right)+\frac{1}{2}\left(\mathrm{G}^{\mathrm{ret}}-\mathrm{G}^{\mathrm{adv}}\right),
$$

where $\mathrm{G}^{\mathrm{adv}}$ is the advanced Green's function. The first term is time symmetric. Namely, under the transformation $t$ $\rightarrow-t$ the retarded field transforms to the advanced field and vice versa, such that the first term is transformed into itself. Because it is time symmetric, the first term does not include the radiative part of the field. Instead, it relates to the nonradiative Coulomb piece of the field, which is the source for the divergence. Dirac then showed that if one integrates only the second term, namely the radiative Green's function, one can obtain an expression for the field of the charge, which is finite on the world line of the charge. One can then use the usual Lorentz force formula to obtain the equation of motion which includes also the radiation reaction effects, i.e., the relativistic Abraham-Lorentz-Dirac equation. Dirac's prescription for regularization of the self force is quite successful for arbitrary motion in flat space-time. However, because it involves the acausal advanced Green's function, it suffers from an inherent difficulty when one wishes to apply it for motion in a gravitational field: In curved space-time the Green's function has support not only on the light cone, but also inside it. In particular, the instantaneous forces on the charge depend on the future history of the charge's motion, which is anticausal. ${ }^{8}$ Calculation of the self force which uses only the causal retarded field should therefore be preferred.

Recently, Ori proposed a direct method for the calculation of self forces based on the retarded Green's function. ${ }^{8,9}$ The 
method is based on the decomposition of the field and the self force into Fourier-harmonic modes. This approach has two important advantages: First, when one decomposes the field into modes one can separate the variables and obtain an ordinary differential equation rather than a partial one. Second, each mode of the self force turns out to be finite (even for pointlike particles), such that divergences (when they occur) are met only when one sums over all modes. The treatment of the individual modes is free from divergences. This approach has the advantages of being both local (such that it does not depend on balance arguments, which are useless for many interesting cases) and causal (such that it does not suffer from the acausalities of the radiative Green's function). Of course, in general, the sum over all modes of the self force diverges, and a regularization prescription is needed. Such a prescription has been recently proposed by Ori. ${ }^{10}$ The availability of a regularization prescription strengthens the motivation for studying the self force using this approach. For many interesting cases the self force (or some of its components) is found to be finite without any need of a regularization procedure. These cases may serve as an exposition of the direct calculation of the self force, without the complications which are involved with regularization techniques.

As an example of the application of this direct method for the calculation of radiation reaction, we present in this paper an analysis of the radiation-reaction force for (electromagnetic) synchrotron radiation. Radiation reaction for this case turns out to be susceptible to an exact analytical calculation, i.e., one can sum analytically over all the Fourier-harmonic modes and obtain a closed-form expression for the self force. In addition, the temporal component of the self force for synchrotron radiation, whose calculation is our main objective in this paper, turns out to be finite, without any need of regularization. In fact, the angular components of the self force also turn out to be finite: it is only the radial component which is divergent. (This divergence can be removed by simple procedures, and the renormalized radial self-force is zero.) The separation of the divergent component of the self force from the finite components is very useful, ${ }^{11}$ as it allows the consideration of important aspects of the self force without getting involved in complicated regularization techniques. (In Sec. VIII we shall comment in passing on the radial component of the self force.)

Synchrotron radiation is an invaluable tool in the understanding of many physical phenomena, and is also very useful in many other fields, such as astronomy, chemistry, biology, medical science, and technology. For recent reviews see, e.g., Refs. 12-14. The importance of synchrotron radiation stems from its unique properties, e.g., its spectral distribution in a broad range of wavelengths, its relatively high intensity compared with other sources, the high degree of directionality of the radiation very close to the plane of the charge's orbit, and its well-defined polarization.

The usual approach to radiation reaction from synchrotron radiation is to compute the far field in the radiation zone, then find the asymptotic expression for the Poynting vector and finally calculate the radiated power by integrating over a large sphere in the radiation zone. Then one expects the radiated power at infinity to equal the rate of loss of energy by the orbiting charge. Specifically, one assumes energy balance, and equates the radiated energy at infinity with the loss of energy by the charge. This approach appears in many standard textbooks, e.g., in Refs. 15 and 16. Here we develop a different approach, which is based on the near field rather than on the far field. Specifically, we consider the electromagnetic field which the orbiting charge produces and find the interaction of this field with the very same charge that created it. Thereby, we construe the loss of energy by the charge as a self force or a back-reaction force. Our result here agrees with the well-known result, and also with the Abraham-Lorentz-Dirac equation. ${ }^{4}$ Our method of calculation yields another important result, which is the multipole decomposition of the radiation. The radiation has been decomposed in the past into Fourier modes, but no previous multipole decomposition is known to us.

We note that our entire discussion here is classical and that we do not consider quantum corrections here. There are known cases where quantum corrections are extremely important, e.g., when there are extremely strong magnetic fields, such as those which exist in magnetars. The importance of quantum corrections is discussed in Ref. 17. However, for most cases the quantum corrections for synchrotron radiation are unimportant, so that a purely classical discussion is useful. We stress that the agreement we find with the known expressions for synchrotron radiation or with the Abraham-Lorentz-Dirac equation is found just because we do not consider quantum correction. The AbrahamLorentz-Dirac equation is purely classical, and does not include any quantum corrections.

Another point which is worth emphasizing here is that our main result, the radiated power for synchrotron radiation, can be obtained very easily using conventional techniques, such as those which appear in Refs. 15 and 16. Our derivation here is much more complicated from the technical point of view. However, from the conceptual point of view it is simple, as it is, in fact, only a direct calculation of the Lorentz force which uses only retarded fields. The technical complication is an unavoidable price which has to be paid in order to use this direct approach.

We also note that the mathematical formalism which we use is more sophisticated than the formalism which is usually used for the treatment of synchrotron radiation. The reason for this is that we consider the electromagnetic field as derived from a four-vector potential. The decomposition of the four-vector potential into Fourier-harmonic modes is possible only in terms of vector spherical harmonics. Vector spherical harmonics are frequently used in standard texts (e.g., in Ref. 15). However, we preferred to use the conventions of indicial vector (and tensor) analysis, rather than the notation of Ref. 15. Because the analysis is done in curved coordinates, we shall frequently use concepts from Riemannian geometry (or pseudo-Riemannian, in a strict mathematical sense). The reader is referred to any text on general relativity, e.g., Ref. 18.

The organization of this paper is as follows. We begin in Sec. II by decomposing the electromagnetic vector potential into vector spherical harmonics. In Appendix A we briefly review the main properties of vector spherical harmonics which are important to us. More details can be found, e.g., in Ref. 19. In Sec. III we compute the electromagnetic field from the vector potential, evaluate the field at the instantaneous position of the charge, and calculate the temporal component of the Lorentz four-force. We shall show that it is this component which is relevant for the radiated power. In Sec. IV we use the more conventional approach and decompose the radiated power at infinity into spherical harmonics. This is done by evaluating the asymptotic far-field electro- 
magnetic field, building the energy-momentum tensor from this field, and integrating the energy flux over a large sphere. This yields the radiated power at infinity directly, and we show that this far-field calculation agrees with the self force approach mode by mode. In Sec. V we collect the contributions from the various multipole moments, and expand the radiated power in a power series. This series can be summed over all orders, and the result we obtain for the total radiated power in Sec. VI coincides with the well-known result, which is obtained, e.g., in Refs. 15 and 16 , by completely different procedures. We demonstrate agreement with the Abraham-Lorentz-Dirac equation in Sec. VII, and discuss our method and results in Sec. VIII.

\section{THE ELECTROMAGNETIC VECTOR POTENTIAL}

Let us consider a pointlike electric charge $q$ in circular orbit in Minkowski space-time, such that the coordinates of the charge, in spherical coordinates, are $r=r_{0}, \theta=\pi / 2, \phi$ $=\phi(t)$, and $t$. The coordinates $r, \theta, \phi, t$ are inertial coordinates, e.g., coordinates of an inertial observer at $r \gg r_{0}$. The proper time of the charge is $\tau$, such that $t=\gamma \tau, \gamma$ being the Lorentz factor. We assume the motion of the charge is uniform, $\phi(\tau)=\gamma \bar{\omega} \tau$. The angular velocity, as measured by a far away inertial observer, is $\bar{\omega}$. We take the metric signature to be $(-+++)$.

\section{A. Temporal component of the vector potential}

We separate the four-vector potential $A^{\mu}$ into a threevector $\mathbf{A}$ and a scalar potential $A^{t}$. Bold-faced quantities denote three-vectors in any basis, namely either in a covariant, contravariant, or normalized basis. (See Appendix A.) We shall use the Lorenz ${ }^{20}$ gauge throughout, ${ }^{21}$ and use units where the speed of light $c=1$. The scalar potential satisfies the inhomogeneous wave equation

$$
\square A^{t}=-4 \pi j^{t},
$$

where the temporal component of the four-current density $j^{t}$ is given by

$$
j^{t}=\rho u^{t}=q \gamma \int_{-\infty}^{\infty} d \tau \frac{\delta^{4}\left[x^{\mu}-x_{0}^{\mu}(\tau)\right]}{r^{2} \sin \theta} .
$$

Here, $\rho$ is the charge density and $u^{t}$ is the temporal component of the four-velocity. We denote by $\square$ the d'Alembertian wave operator. Decomposition of $j^{t}$ into Fourier-harmonic modes yields

$$
\begin{aligned}
j^{t}= & q \frac{\delta\left(r-r_{0}\right)}{r_{0}^{2}} \int_{\omega=-\infty}^{\infty} d \omega e^{-i \omega t} \\
& \times \sum_{l m} \delta(\omega-m \bar{\omega}) \bar{P}^{l m}(0) Y^{l m}(\theta, \phi) .
\end{aligned}
$$

We denote $\bar{P}^{l m}(0) \equiv e^{-i m \phi} Y^{l m}(\cos \theta=0, \phi)$. In the Fourier decomposition we take the frequencies of the Fourier modes to be both positive and negative. We next decompose the scalar potential into modes according to

$$
A^{t}=\sum_{l m} \int_{\omega=-\infty}^{\infty} d \omega \frac{1}{r} \psi_{l m, \omega}(r) Y^{l m}(\theta, \phi) e^{-i \omega t} .
$$

Then, the left-hand side (lhs) of the wave equation becomes

$$
\begin{aligned}
\square A^{t}= & \sum_{l m} \int_{\omega=-\infty}^{\infty} d \omega\left\{\frac{1}{r} \omega_{l m, \omega}^{\prime \prime}\right. \\
& \left.+\left[\omega^{2}-\frac{l(l+1)}{r^{2}}\right] \frac{1}{r} \omega_{l m, \omega}\right\} Y^{l m} e^{-i \omega t} .
\end{aligned}
$$

A prime denotes differentiation with respect to the radial coordinate $r$. Substituting into the wave equation (1) we find for the modes of the scalar potential

$$
\begin{aligned}
\psi_{l m, \omega}^{\prime \prime} & +\left[\omega^{2}-\frac{l(l+1)}{r^{2}}\right] \psi_{l m, \omega} \\
= & -4 \pi q \frac{\delta\left(r-r_{0}\right)}{r_{0}} \delta(\omega-m \bar{\omega}) \bar{P}^{l m}(0) .
\end{aligned}
$$

We choose the solutions of the corresponding homogeneous equation to be such that one solution is regular at the origin (namely, at $r=0$ ), and the other solution describes purely outgoing modes at infinity. These requirements are equivalent to choosing a retarded solution, which is the physically relevant solution. These basic functions which solve the homogeneous equation are

$$
\psi_{l}^{0}=\sqrt{\frac{|\omega|}{2 \pi}} r j^{l}(\omega r)
$$

and

$$
\psi_{l}^{\infty}=\sqrt{\frac{|\omega|}{2 \pi}} r h^{l}(\omega r),
$$

where $j^{l}$ and $h^{l}$ are the spherical Bessel and Hankel functions of the first kinds, respectively. Our choice of normalization (which is, of course, not determined from the homogeneous equations) is the same as in Ref. 19. The Wronskian determinant ${ }^{22}$ of these two solutions is $\mathrm{W}=i \operatorname{sgn}(\omega) /(2 \pi)$. We show in Appendix B that the solution for Eq. (5) can be written as a sum of two terms, each proportional to a Heaviside step function. Specifically, the $I m$ mode of the scalar potential is given by

$$
\begin{aligned}
A_{l m}^{t}= & \frac{f\left(r_{0}\right) \psi_{l m}^{\infty}\left(r_{0}\right)}{W} \frac{1}{r} \psi_{l m}^{0}(r) e^{-i \omega t} Y^{l m}(\theta, \phi) \Theta\left(r_{0}-r\right) \\
& +\frac{f\left(r_{0}\right) \psi_{l m}^{0}\left(r_{0}\right)}{W} \frac{1}{r} \psi_{l m}^{\infty}(r) e^{-i \omega t} Y^{l m}(\theta, \phi) \Theta\left(r-r_{0}\right),
\end{aligned}
$$

where $\Theta(x)=1$ for $x>0$ and $\Theta(x)=0$ for $x<0$ is the Heaviside step function, and where $f(r)=-(4 \pi q / r) \delta(\omega$ $-m \bar{\omega}) \bar{P}^{l m}(0)$.

\section{B. Spatial components of the vector potential}

The vector potential A satisfies, in the Lorenz gauge, an inhomogeneous wave equation

$$
\square \mathbf{A}=-4 \pi \mathbf{j},
$$

where $\mathbf{j}=\rho \mathbf{u}$ is the three-current density of the sources for the field, and where $\mathbf{u}$ is the three-velocity. Decomposing the 
current density $\mathbf{j}$ into vector harmonics, we find

$$
\begin{aligned}
j_{l}^{\wedge}= & q \bar{\omega} \frac{\delta\left(r-r_{0}\right)}{r_{0}} \int_{\omega=-\infty}^{\infty} d \omega e^{-i \omega t} \\
& \times \sum_{l^{\prime}, l m} \delta(\omega-m \bar{\omega}) \bar{P}_{\hat{\phi}}^{l^{\prime}, l m}(0) Y_{i}^{l^{\prime}, l m}(\theta, \phi),
\end{aligned}
$$

where $\bar{P}_{\hat{\phi}}^{l^{\prime}, l m}(0)=e^{-i m \phi} Y_{\hat{\phi}}^{l^{\prime}, l m}(\cos \theta=0, \phi)$. We denote by a coordinate with an over-hat the coordinate in a normalized basis (see Appendix A), and by a star we denote complex conjugation. By $Y_{i}^{l^{\prime}}, l m$ we denote a component in a normalized basis of the pure-orbital vector spherical harmonics (see Appendix A). We next decompose the vector potential into modes according to

$A_{\hat{\imath}}=\sum_{l^{\prime}, l m} \int_{\omega=-\infty}^{\infty} d \omega \frac{1}{r} \Psi_{l^{\prime}, l m, \omega}(r) Y_{\hat{\imath}}^{l^{\prime}, l m}(\theta, \phi) e^{-i \omega t}$.

Then, the decomposition of the lhs of the wave equation into modes yields

$$
\begin{aligned}
\square A_{\imath}= & \sum_{l^{\prime}, l m} \int_{\omega=-\infty}^{\infty} d \omega\left\{\frac{1}{r} \Psi_{l^{\prime}, l m, \omega}^{\prime \prime}\right. \\
& \left.+\left[\omega^{2}-\frac{l^{\prime}\left(l^{\prime}+1\right)}{r^{2}}\right] \frac{1}{r} \Psi_{l^{\prime}, l m, \omega}\right\} Y_{\stackrel{l}{l^{\prime}, l m} e^{-i \omega t} .}
\end{aligned}
$$

Each mode of the vector potential satisfies the equation

$$
\begin{aligned}
& \Psi_{l^{\prime}, l m, \omega}^{\prime \prime}+\left[\omega^{2}-\frac{l^{\prime}\left(l^{\prime}+1\right)}{r^{2}}\right] \Psi_{l^{\prime}, l m, \omega} \\
& \quad=-4 \pi q \bar{\omega} \delta\left(r-r_{0}\right) \delta(\omega-m \bar{\omega}) \bar{P}_{\hat{\phi}}^{l^{\prime}, l m^{*}}(0) .
\end{aligned}
$$

The basic solutions of the corresponding homogeneous equation are

$$
\Psi_{l^{\prime}}^{0}=\sqrt{\frac{|\omega|}{2 \pi}} r j^{l^{\prime}}(\omega r)
$$

and

$$
\Psi_{l^{\prime}}^{\infty}=\sqrt{\frac{|\omega|}{2 \pi}} r h^{l^{\prime}}(\omega r)
$$

such that the solution for the $l^{\prime}, l m$ mode of the vector potential takes the form

$$
\begin{aligned}
\mathbf{A}^{l^{\prime}, l m}= & \frac{F\left(r_{0}\right) \Psi_{l^{\prime}}^{\infty}\left(r_{0}\right)}{W} \frac{1}{r} \Psi_{l^{\prime}}^{0}(r) \\
& \times e^{-i \omega t} \mathbf{Y}^{l^{\prime}, l m}(\theta, \phi) \Theta\left(r_{0}-r\right) \\
& +\frac{F\left(r_{0}\right) \Psi_{l^{\prime}}^{0}\left(r_{0}\right)}{W} \frac{1}{r} \Psi_{l^{\prime}}^{\infty}(r) \\
& \times e^{-i \omega t} \mathbf{Y}^{l^{\prime}, l m}(\theta, \phi) \Theta\left(r-r_{0}\right),
\end{aligned}
$$

where $F(r)=-4 \pi q \bar{\omega} \delta(\omega-m \bar{\omega}) \bar{P}_{\hat{\phi}}^{l^{\prime}, l m^{*}}(0)$.

We find it convenient to separate the electromagnetic field into electric and magnetic modes, which we later find (as indeed should be expected) to propagate on their own without mixing. The index $l^{\prime}$ of the pure-orbital vector harmonics (do not confuse the prime here with differentiation with respect to $r$ ) can take the values $l-1, l$, or $l+1$. After direct substitution of the explicit expressions for the various functions in Eq. (12) and summation over all $l^{\prime}$ modes, we find the $l m$ mode of the magnetic part of the vector potential to be

$$
\begin{aligned}
&{ }^{M} A_{\hat{t}}^{l m}={ }^{M} A_{\hat{r}}^{l m}=0, \\
&{ }^{M} A_{\hat{\theta}}^{l m}=-a_{0}^{l, l m} j^{l}\left(m \bar{\omega} r_{0}\right) \frac{m}{\sqrt{l(l+1)}}\left[h^{l}(m \bar{\omega} r) e^{-i m \bar{\omega} t} \frac{Y^{l m}(\theta, \phi)}{\sin \theta}+\text { c.c. }\right] \Theta\left(r-r_{0}\right) \\
&-a_{0}^{l, l m} j^{l}(m \bar{\omega} r) \frac{m}{\sqrt{l(l+1)}}\left[h^{l}\left(m \bar{\omega} r_{0}\right) e^{-i m \bar{\omega} t} \frac{Y^{l m}(\theta, \phi)}{\sin \theta}+\text { c.c. }\right] \Theta\left(r_{0}-r\right), \\
&{ }^{M} A_{\hat{\phi}}^{l m}=-a_{0}^{l, l m} j^{l}\left(m \bar{\omega} r_{0}\right) \frac{1}{\sqrt{l(l+1)}}\left[i h^{l}(m \bar{\omega} r) e^{-i m \bar{\omega} t} \frac{\partial Y^{l m}(\theta, \phi)}{\partial \theta}+\text { c.c. }\right] \Theta\left(r-r_{0}\right) \\
& \quad-a_{0}^{l, l m} j^{l}(m \bar{\omega} r) \frac{1}{\sqrt{l(l+1)}}\left[i h^{l}\left(m \bar{\omega} r_{0}\right) e^{-i m \bar{\omega} t} \frac{\partial Y^{l m}(\theta, \phi)}{\partial \theta}+\text { c.c. }\right] \Theta\left(r_{0}-r\right),
\end{aligned}
$$

and, similarly, for the electric part

$$
\begin{aligned}
{ }^{E} A_{\hat{t}}^{l m}= & \varphi_{0} j^{l}\left(m \bar{\omega} r_{0}\right)\left[i h^{l}(m \bar{\omega} r) e^{-i m \bar{\omega} t} Y^{l m}(\theta, \phi)+\text { c.c. }\right] \Theta\left(r-r_{0}\right) \\
& +\varphi_{0} j^{l}(m \bar{\omega} r)\left[i h^{l}\left(m \bar{\omega} r_{0}\right) e^{-i m \bar{\omega} t} Y^{l m}(\theta, \phi)+\text { c.c. }\right] \Theta\left(r_{0}-r\right),
\end{aligned}
$$




$$
\begin{aligned}
& { }^{E} A_{\hat{r}}^{l m}=\left\{-a_{0}^{l+1, l m} j^{l+1}\left(m \bar{\omega} r_{0}\right) \sqrt{\frac{l+1}{2 l+1}}\left[h^{l+1}(m \bar{\omega} r) e^{-i m \bar{\omega} t} Y^{l m}(\theta, \phi)+\text { c.c. }\right]\right. \\
& \left.+a_{0}^{l-1, l m} j^{l-1}\left(m \bar{\omega} r_{0}\right) \sqrt{\frac{l}{2 l+1}}\left[h^{l-1}(m \bar{\omega} r) e^{-i m \bar{\omega} t} Y^{l m}(\theta, \phi)+\text { c.c. }\right]\right\} \Theta\left(r-r_{0}\right) \\
& +\left\{-a_{0}^{l+1, l m} j^{l+1}(m \bar{\omega} r) \sqrt{\frac{l+1}{2 l+1}}\left[h^{l+1}\left(m \bar{\omega} r_{0}\right) e^{-i m \bar{\omega} t} Y^{l m}(\theta, \phi)+\text { c.c. }\right]\right. \\
& \left.+a_{0}^{l-1, l m} j^{l-1}(m \bar{\omega} r) \sqrt{\frac{l}{2 l+1}}\left[h^{l-1}\left(m \bar{\omega} r_{0}\right) e^{-i m \bar{\omega} t} Y^{l m}(\theta, \phi)+\text { c.c. }\right]\right\} \Theta\left(r_{0}-r\right), \\
& { }^{E} A_{\hat{\phi}}^{l m}=\left\{a_{0}^{l+1, l m} j^{l+1}\left(m \bar{\omega} r_{0}\right) \frac{m}{\sqrt{(l+1)(2 l+1)}}\left[i h^{l+1}(m \bar{\omega} r) e^{-i m \bar{\omega} t} \frac{Y^{l m}(\theta, \phi)}{\sin \theta}+\text { c.c. }\right]\right. \\
& \left.+a_{0}^{l-1, l m} j^{l-1}\left(m \bar{\omega} r_{0}\right) \frac{m}{\sqrt{l(2 l+1)}}\left[i h^{l-1}(m \bar{\omega} r) e^{-i m \bar{\omega} t} \frac{Y^{l m}(\theta, \phi)}{\sin \theta}+\text { c.c. }\right]\right\} \Theta\left(r-r_{0}\right) \\
& +\left\{a_{0}^{l+1, l m} j^{l+1}(m \bar{\omega} r) \frac{m}{\sqrt{(l+1)(2 l+1)}}\left[i h^{l+1}\left(m \bar{\omega} r_{0}\right) e^{-i m \bar{\omega} t} \frac{Y^{l m}(\theta, \phi)}{\sin \theta}+\text { c.c. }\right]\right. \\
& \left.+a_{0}^{l-1, l m} j^{l-1}(m \bar{\omega} r) \frac{m}{\sqrt{l(2 l+1)}}\left[i h^{l-1}\left(m \bar{\omega} r_{0}\right) e^{-i m \bar{\omega} t} \frac{Y^{l m}(\theta, \phi)}{\sin \theta}+\text { c.c. }\right]\right\} \Theta\left(r_{0}-r\right), \\
& { }^{E} A_{\hat{\theta}}^{l m}=\left\{a_{0}^{l+1, l m} j^{l+1}\left(m \bar{\omega} r_{0}\right) \frac{1}{\sqrt{(l+1)(2 l+1)}}\left[h^{l+1}(m \bar{\omega} r) e^{-i m \bar{\omega} t} \frac{\partial Y^{l m}(\theta, \phi)}{\partial \theta}+\text { c.c. }\right]\right. \\
& \left.+a_{0}^{l-1, l m} j^{l-1}\left(m \bar{\omega} r_{0}\right) \frac{1}{\sqrt{l(2 l+1)}}\left[h^{l-1}(m \bar{\omega} r) e^{-i m \bar{\omega} t} \frac{\partial Y^{l m}(\theta, \phi)}{\partial \theta}+\text { c.c. }\right]\right\} \Theta\left(r-r_{0}\right) \\
& +\left\{a_{0}^{l+1, l m} j^{l+1}(m \bar{\omega} r) \frac{1}{\sqrt{(l+1)(2 l+1)}}\left[h^{l+1}\left(m \bar{\omega} r_{0}\right) e^{-i m \bar{\omega} t} \frac{\partial Y^{l m}(\theta, \phi)}{\partial \theta}+\text { c.c. }\right]\right. \\
& \left.+a_{0}^{l-1, l m} j^{l-1}(m \bar{\omega} r) \frac{1}{\sqrt{l(2 l+1)}}\left[h^{l-1}\left(m \bar{\omega} r_{0}\right) e^{-i m \bar{\omega} t} \frac{\partial Y^{l m}(\theta, \phi)}{\partial \theta}+\text { c.c. }\right]\right\} \Theta\left(r_{0}-r\right) \text {. }
\end{aligned}
$$

Here,

$$
\begin{aligned}
& a_{0}^{l+1, l m}=\frac{4 \pi q m^{2} \bar{\omega}^{2} r_{0}}{\sqrt{(l+1)(2 l+1)}} \bar{P}^{l m}(0), \\
& a_{0}^{l, l m}=-\frac{4 \pi q m \bar{\omega}^{2} r_{0}}{\sqrt{l(l+1)}} \frac{\partial \bar{P}^{l m}(0)}{\partial \theta}, \\
& a_{0}^{l-1, l m}=\frac{4 \pi q m^{2} \bar{\omega}^{2} r_{0}}{\sqrt{l(2 l+1)}} \bar{P}^{l m}(0), \\
& \varphi_{0}=-4 \pi q m \bar{\omega} \bar{P}^{l m}(0),
\end{aligned}
$$

where $\partial \bar{P}^{l m}(0) / \partial \theta=\partial Y^{l m} / \partial \theta(\theta=\pi / 2, \phi=0)$. The full vector potential is obtained by summing over the electric and the magnetic contributions, i.e., by $A_{\hat{\mu}}^{l m}={ }^{E} A_{\hat{\mu}}^{l m}+{ }^{M} A_{\hat{\mu}}^{l m}$. By taking the complex conjugate of each component of the vector potential, we summed, in fact, each $m$ with its reciprocal negative counterpart. Specifically, because complex conjugation is equivalent to the transformation $m \rightarrow-m$, adding the complex conjugate is equivalent to adding the $-m$ counter- part to each term. Consequently, when we sum below over all modes, we need only to sum over positive values of $m$.

We shall need below the following explicit expressions, which are easy to derive: ${ }^{23}$

$$
\begin{aligned}
\bar{P}^{l m}(0)= & (-1)^{m} \sqrt{\frac{2 l+1}{4 \pi}} \frac{\sqrt{(l-m) !(l+m) !}}{2^{l}[(l-m) / 2] ![(l+m) / 2] !} \\
& \times \cos \left[\frac{\pi}{2}(l-m)\right],
\end{aligned}
$$

$$
\begin{aligned}
\frac{\partial \bar{P}^{l m}}{\partial \theta}(0)= & (-1)^{m+1} \\
& \times \sqrt{\frac{2 l+1}{4 \pi}} \frac{\sqrt{(l-m) !(l+m+1) !(l+m+1)}}{2^{l}[(l-m-1) / 2] ![(l+m+1) / 2] !} \\
& \times \sin \left[\frac{\pi}{2}(l-m)\right] .
\end{aligned}
$$




\section{The $\omega=0$ modes}

We have not considered the possibility that $\omega=0$. This case corresponds to the magnetic number $m=0$. In this case the terms proportional to $\omega^{2}$ on the lhs of the wave equations (1) and (7) no longer appear. The independent solutions of the homogeneous equations are now $\psi_{0}^{0}=r^{l+1}, \psi_{0}^{\infty}=r^{-l}$, $\Psi_{0}^{0}=r^{l^{\prime}+1}$, and $\Psi_{0}^{\infty}=r^{-l^{\prime}}$. The Wronskian determinants are $\mathrm{W}=2 l+1$ and $\mathrm{W}=2 l^{\prime}+1$, respectively.

We find the vector potential for this case to be

$$
\begin{aligned}
A^{\hat{t} l m}= & -\frac{4 \pi q \bar{P}^{l m}(0)}{2 l+1} \frac{r_{0}^{l}}{r^{l+1}} Y^{l m} \Theta\left(r-r_{0}\right) \\
& -\frac{4 \pi q \bar{P}^{l m}(0)}{2 l+1} \frac{r^{l}}{r_{0}^{l+1}} Y^{l m} \Theta\left(r_{0}-r\right), \\
\mathbf{A}^{l^{\prime}, l m}= & -\frac{4 \pi q \bar{P}_{\hat{\phi}}^{l^{\prime}, l m}(0)}{2 l^{\prime}+1} \frac{r_{0}^{l^{\prime}+1}}{r^{l^{\prime}+1}} \mathbf{Y}^{l^{\prime}, l m} \Theta\left(r_{0}-r\right) \\
& -\frac{4 \pi q \bar{P}_{\hat{\phi}}^{l^{\prime}, l m}(0)}{2 l^{\prime}+1} \frac{r^{l^{\prime}+1}}{r_{0}^{l^{\prime}+1}} \mathbf{Y}^{l^{l^{\prime}, l m} \Theta\left(r-r_{0}\right) .}
\end{aligned}
$$

It can be readily shown that this four-vector potential does not contribute to the temporal component of the self force or to the power carried off by the radiation, so we shall ignore it in the sequel.

\section{THE SELF FORCE}

We wish to present a calculation of the radiation-reaction force which is based on the near field of the charge. We first calculate the temporal component of the Lorentz force, namely, $f^{\hat{t}}=q F^{\hat{t} \hat{\mu}} u_{\hat{\mu}}$, where $u_{\mu}$ is the four-velocity of the charge $q$, and $F_{\alpha \beta}$ is the Maxwell field-strength tensor which is produced by the charge itself. This expression for $f^{\hat{t}}$ is then to be evaluated on the world-line of the charge. The most convenient way to calculate the components of the Maxwell tensor is in covariant components, because then we have simply $F_{\mu \nu}=A_{\nu, \mu}-A_{\mu, \nu}$, and we do not have to worry about covariant differentiation. (Although the metric is flat, the spherical coordinates we use are curved, and therefore one would, in general, need to use covariant derivatives rather than partial derivatives.) Then, we shall change bases again to a normalized basis. We note that $A_{\phi}=r \sin \theta A_{\hat{\phi}}$, $A_{\theta}=r A_{\hat{\theta}}, A_{r}=A_{\hat{r}}$, and $A_{t}=A_{\hat{t}}$.

After calculating the temporal component of the self fourforce we shall also calculate the radiated power, in order to demonstrate their compatibility. We shall therefore be interested below in the radiated power, specifically in $-d E^{l m} / d t$, where $E$ is the energy of the charge. That is, $d E / d t$ is the rate of change of the charge's energy, which is negative because the charge loses energy by radiating. The radiated power $\mathcal{P}$ is positive, and by conservation of energy $\mathcal{P}=-d E / d t$. Spe- cifically, $-d E / d t=\int T^{t \mu} d s_{\mu}=\int T^{t r} d s_{r}$, taking the surface of integration to be a large sphere whose normal is radial. Then, $d s_{\hat{r}}=r^{2} d \Omega$, where $d \Omega=\sin \theta d \theta d \phi$. That is, we take for the radiated power at infinity $-d E^{l m} / d t=\int T^{\hat{t} \hat{r} l m} r^{2} d \Omega$, where $T_{\mu \nu}$ is the energy-momentum tensor of the electromagnetic field. However, the temporal component of the self four-force, by Newton's law, is the rate of change of the temporal component of the four-momentum, namely, the time derivative of the energy. In what follows we shall calculate both $f^{\hat{t}}$ on the charge's world-line and the radiated power at infinity, and show that they agree.

\section{A. Magnetic modes}

The only nonvanishing components of the four velocity are the temporal and the azimuthal components. Namely, only $u_{t}$ and $u_{\phi}$ are not zero. Because the Maxwell tensor is skew-symmetric, it is clear that only $F^{t \phi}$ can contribute to the temporal component of the Lorentz force. Calculation of this component yields

$$
\begin{aligned}
{ }^{M} F_{t \phi}^{l m}= & { }^{M} A_{\phi, t}^{l m}-{ }^{M} A_{t, \phi}^{l m}={ }^{M} A_{\phi, t}^{l m}=r \sin \theta^{M} A_{\hat{\phi}, t}^{l m} \\
= & -m \bar{\omega} a_{0}^{l, l m} r \sin \theta j^{l}\left(m \bar{\omega} r_{0}\right) \\
& \times\left[i h^{l}(m \bar{\omega} r) e^{-i m \bar{\omega} t} Y_{\hat{\phi}}^{l, l m}+\text { c.c. }\right] \Theta\left(r-r_{0}\right) \\
& -m \bar{\omega} a_{0}^{l, l m} r \sin \theta j^{l}(m \bar{\omega} r) \\
& \times\left[i h^{l}\left(m \bar{\omega} r_{0}\right) e^{-i m \bar{\omega} t} Y_{\hat{\phi}}^{l, l m}+\text { c.c. }\right] \Theta\left(r_{0}-r\right) .
\end{aligned}
$$

When this is evaluated on the charge's world-line, we find that the field is continuous at $r_{0}$. (We find that to be the case, in fact, for the entire temporal component of the self fourforce.) We thus find that on the charge's world line

$$
\left.{ }^{M_{F}} F_{\hat{t} \hat{\phi}}^{l m}\right|_{\text {charge }}=-2 \frac{m \bar{\omega}}{\sqrt{l(l+1)}} a_{0}^{l, l m} j^{l^{2}}\left(m \bar{\omega} r_{0}\right) \frac{\partial \bar{P}^{l m}}{\partial \theta}(0) .
$$

Then, the contribution of the magnetic modes to the temporal component of the self force is given by

$$
\begin{aligned}
\left.{ }^{M} f^{\hat{t}, l m}\right|_{\text {charge }} & =\left.{ }^{M} F^{\hat{t} \hat{\phi}, l m} u_{\hat{\phi}}\right|_{\text {charge }} \\
& =2 q \gamma r_{0} \frac{m \bar{\omega}^{2}}{\sqrt{l(l+1)}} a_{0}^{l, l m} j^{l^{2}}\left(m \bar{\omega} r_{0}\right) \frac{\partial \bar{P}^{l m}}{\partial \theta}(0),
\end{aligned}
$$

where $\left.u_{\hat{\phi}}\right|_{\text {charge }}=r_{0} \bar{\omega} \gamma$.

\section{B. Electric modes}

Contributions to the electric modes come from $l^{\prime}=l+1$ and $l^{\prime}=l-1$. We denote the sum over these two values of $l^{\prime}$ by $\bar{l}$ :

$$
{ }^{E} A_{\hat{\phi}}^{\bar{l}, l m}={ }^{E} A_{\hat{\phi}}^{l+1, l m}+{ }^{E} A_{\hat{\phi}}^{l-1, l m}=\left\{\frac{m}{\sqrt{(l+1)(2 l+1)}} a_{0}^{l+1, l m} j^{l+1}\left(m \bar{\omega} r_{0}\right)\left[i h^{l+1}(m \bar{\omega} r) e^{-i m \bar{\omega} t} \frac{Y^{l m}}{\sin \theta}+\text { c.c. }\right]\right.
$$




$$
\begin{aligned}
& \left.+\frac{m}{\sqrt{l(2 l+1)}} a_{0}^{l-1, l m} j^{l-1}\left(m \bar{\omega} r_{0}\right)\left[i h^{l-1}(m \bar{\omega} r) e^{-i m \bar{\omega} t} \frac{Y^{l m}}{\sin \theta}+\text { c.c. }\right]\right\} \Theta\left(r-r_{0}\right) \\
& +\left\{\frac{m}{\sqrt{(l+1)(2 l+1)}} a_{0}^{l+1, l m} j^{l+1}(m \bar{\omega} r)\left[i h^{l+1}\left(m \bar{\omega} r_{0}\right) e^{-i m \bar{\omega} t} \frac{Y^{l m}}{\sin \theta}+\text { c.c. }\right]\right. \\
& \left.+\frac{m}{\sqrt{l(2 l+1)}} a_{0}^{l-1, l m} j^{l-1}(m \bar{\omega} r)\left[i h^{l-1}\left(m \bar{\omega} r_{0}\right) e^{-i m \bar{\omega} t} \frac{Y^{l m}}{\sin \theta}+\text { c.c. }\right]\right\} \Theta\left(r_{0}-r\right),
\end{aligned}
$$

such that the $t \phi$ covariant component of the Maxwell tensor is

$$
\begin{aligned}
\left.{ }^{E} F_{t \phi}^{l m}\right|_{\text {charge }}= & { }^{E} A_{\phi, t}^{\bar{l}, l m}-\left.{ }^{E} A_{t, \phi}^{l, l m}\right|_{\text {charge }} \\
= & 2 a_{0}^{l+1, l m} \frac{m^{2} \bar{\omega} r_{0}}{\sqrt{(l+1)(2 l+1)}} j^{l+1^{2}}\left(m \bar{\omega} r_{0}\right) \bar{P}^{l m}(0) \\
& +2 a_{0}^{l-1, l m} \frac{m^{2} \bar{\omega} r_{0}}{\sqrt{l(2 l+1)}} j^{l-1^{2}}\left(m \bar{\omega} r_{0}\right) \bar{P}^{l m}(0) \\
& +2 \varphi_{0} m j^{l^{2}}\left(m \bar{\omega} r_{0}\right) \bar{P}^{l m}(0) \\
= & \frac{1}{2 \pi q \bar{\omega}}\left\{\left[a_{0}^{l+1, l m} j^{l+1}\left(m \bar{\omega} r_{0}\right)\right]^{2}\right. \\
& \left.+\left[a_{0}^{l-1, l m} j^{l-1}\left(m \bar{\omega} r_{0}\right)\right]^{2}-\left[\varphi_{0} j^{l}\left(m \bar{\omega} r_{0}\right)\right]^{2}\right\},
\end{aligned}
$$

and the contribution of the electric modes to the temporal component of the self force is given by

$$
\begin{aligned}
\left.{ }^{E} f^{\hat{t}, l m}\right|_{\text {charge }}= & \left.q^{E} F^{\hat{t} \hat{\phi}, l m} u_{\hat{\phi}}\right|_{\text {charge }} \\
= & -8 \pi \gamma q^{2} m^{4} \bar{\omega}^{4} r_{0}^{2} \bar{P}^{l m} 2(0) \frac{l(l+1)}{(2 l+1)^{2}} \\
& \times\left[\frac{1}{l+1} j^{l+1}\left(m \bar{\omega} r_{0}\right)-\frac{1}{l} j^{l-1}\left(m \bar{\omega} r_{0}\right)\right]^{2} .
\end{aligned}
$$

\section{The total force}

Collecting the contributions of the magnetic and electric modes we find that the $l m$ mode of $f^{\hat{t}}$ is

$$
\left.f^{\hat{t}, l m}\right|_{\text {charge }}=-8 \pi \gamma\left(q m^{2} \bar{\omega}^{2} r_{0}\right)^{2} l(l+1) \mathcal{T}^{l m},
$$

where

$$
\mathcal{T}^{l m}=\left[\frac{\bar{P}^{l m}(0) X^{l m}}{2 l+1}\right]^{2}+\left[\frac{1}{m l(l+1)} \frac{\partial \bar{P}^{l m}}{\partial \theta}(0) j^{l}\left(m \bar{\omega} r_{0}\right)\right]^{2} .
$$

Here,

$$
X^{l m}=\frac{1}{l} j^{l-1}\left(m \bar{\omega} r_{0}\right)-\frac{1}{l+1} j^{l+1}\left(m \bar{\omega} r_{0}\right) .
$$

In what follows we shall show that this expression coincides with the radiated power at infinity, and expand the force into a power series, which will enable us to find out how much radiation there is in each multipole moment.

\section{MODE DECOMPOSITION OF THE RADIATED POWER AT INFINITY}

We next expand the Maxwell tensor about $r=\infty$, and calculate the leading-order term in $r^{-1}$ of the $t r$ component of the energy-momentum tensor, which is related to the radiated power. In each term of the energy-momentum tensor there is a product of two components of the Maxwell tensor. It can be shown that these products do not mix fields of different parities or components of different multipole moments. Namely, magnetic modes do not mix with electric modes (as should indeed be expected), and modes of different values of $l$ or $m$ do not mix. By a right arrow we denote that the quantity on the left-hand side approaches the expression on the right-hand side as $r$ approaches infinity. We find

$$
\begin{aligned}
{ }^{M} F_{\hat{t} \hat{\phi}}^{l m},{ }^{M} F_{\hat{\phi} \hat{r}}^{l m} \rightarrow & (-1)^{l} i^{l+1} a_{0}^{l, l m} j^{l}\left(m \bar{\omega} r_{0}\right) \\
& \times \frac{1}{\sqrt{l(l+1)}} \frac{1}{r} \frac{\partial Y^{l m}}{\partial \theta} e^{i m \bar{\omega}(r-t)}+\text { c.c. }
\end{aligned}
$$

such that

$$
\begin{aligned}
{ }^{M} F_{\hat{t} \hat{\phi}}^{l m}{ }^{M} F^{\hat{\phi} \hat{r}, l m} \rightarrow & 2 a_{0}^{l, l m 2} \frac{1}{l(l+1)} \frac{1}{r^{2}} j^{l^{2}}\left(m \bar{\omega} r_{0}\right) \\
& \times \frac{\partial Y^{l m}}{\partial \theta} \frac{\partial Y^{l m *}}{\partial \theta}+\text { o.t. }
\end{aligned}
$$

We denote by o.t. oscillatory terms, whose contribution to the total radiated power vanishes after integration over the sphere. Similarly,

${ }^{M} F_{\hat{t} \hat{\theta}}^{l m},{ }^{M} F_{\hat{\theta} \hat{r}}^{l m} \rightarrow(-i)^{l} a_{0}^{l, l m} j^{l}(m \bar{\omega} r)$

$$
\times \frac{m}{\sqrt{l(l+1)}} \frac{1}{r} \frac{Y^{l m}}{\sin \theta} e^{i m \bar{\omega}(r-t)}+\text { c.c. }
$$

such that

$$
\begin{aligned}
{ }^{M} F_{\hat{t} \hat{\theta}}^{l m} M^{M} F^{\hat{\theta} \hat{r}, l m} \rightarrow & 2 a_{0}^{l, l m 2} \frac{m^{2}}{l(l+1)} \frac{1}{r^{2}} j^{l^{2}}\left(m \bar{\omega} r_{0}\right) \\
& \times \frac{Y^{l m} Y^{l m *}}{\sin ^{2} \theta}+\text { o.t. }
\end{aligned}
$$

The total contribution of the magnetic modes to the energymomentum tensor is thus

$$
{ }^{M} T_{\hat{t}}^{\hat{r}, l m}=\frac{1}{4 \pi}\left({ }^{M} F_{\hat{t} \hat{\phi}}^{l m} M F^{\hat{\phi} \hat{r}, l m}+{ }^{M} F_{\hat{t} \hat{\theta}}^{l m} M F^{\hat{\theta} \hat{r}, l m}\right) .
$$

We integrate this component of the energy-momentum tensor over a large sphere at infinity. Recalling that 


$$
\begin{aligned}
& \int\left(\frac{\partial Y^{l m}}{\partial \theta} \frac{\partial Y^{\tilde{l} \tilde{m} *}}{\partial \theta}+\frac{m^{2}}{\sin ^{2} \theta} Y^{l m} Y^{\tilde{l} \tilde{m} *}\right) d \Omega \\
& =l(l+1) \delta_{l \tilde{l}} \delta_{m \tilde{m}},
\end{aligned}
$$

we find

$$
\begin{aligned}
\mathcal{P}^{M l m} & =-\int{ }^{M} T^{\hat{t} \hat{r}, l m} r^{2} d \Omega \\
& =\frac{1}{2 \pi} a_{0}^{l, l m}{ }^{j^{l^{2}}\left(m \bar{\omega} r_{0}\right)} \\
& =8 \pi q^{2} \bar{\omega}^{4} r_{0}^{2} \frac{m^{2}}{l(l+1)} \frac{\partial \bar{P}^{l m}(0)^{2}}{\partial \theta} j^{l^{2}}\left(m \bar{\omega} r_{0}\right) .
\end{aligned}
$$

For the contribution of the electric modes we find

$$
\begin{aligned}
{ }^{E} F_{\hat{t} \hat{\phi}}^{l m},{ }^{E} F_{\hat{\phi} \hat{r}}^{l m} \rightarrow & -(-i) \frac{4 \pi q m^{3} \bar{\omega}^{2}}{r} \frac{r_{0} \bar{P}^{l m}(0)}{2 l+1} \\
& \times X^{l m} \frac{Y^{l m}}{\sin \theta} e^{i m \bar{\omega}(r-t)}+\text { c.c. }
\end{aligned}
$$

and

$$
\begin{aligned}
{ }^{E} F_{\hat{t} \hat{\theta}}^{l m},{ }^{E} F_{\hat{\theta} \hat{r}}^{l m} \rightarrow & -(-i)^{l+1} \frac{4 \pi q m^{2} \bar{\omega}^{2}}{r} \frac{r_{0} \bar{P}^{l m}(0)}{2 l+1} \\
& \times X^{l m} \frac{\partial Y^{l m}}{\partial \theta} e^{i m \bar{\omega}(r-t)}+\text { c.c. }
\end{aligned}
$$

such that

$$
{ }^{E} T_{\hat{t}}^{\hat{r}, l m}=\frac{1}{4 \pi}\left({ }^{E} F_{\hat{t} \hat{\phi}}^{l m} E F^{\hat{\phi} \hat{r}, l m}+{ }^{E} F_{\hat{t} \hat{\theta}}^{l m} E F^{\hat{\theta} \hat{r}, l m}\right),
$$

and we find

$$
\begin{aligned}
\mathcal{P}^{E l m}= & -\int{ }^{E} T^{\hat{t} \hat{r}, l m} r^{2} d \Omega \\
= & 8 \pi q^{2} m^{4} \bar{\omega}^{4} r_{0}^{2} \frac{l(l+1)}{(2 l+1)^{2}} \bar{P}^{l m 2}(0) \\
& \times\left[\frac{1}{l+1} j^{l+1}\left(m \bar{\omega} r_{0}\right)-\frac{1}{l} j^{l-1}\left(m \bar{\omega} r_{0}\right)\right]^{2} .
\end{aligned}
$$

The total radiated power for the $\mathrm{lm}$ modes is the sum of the magnetic and the electric contributions, which coincides with the expression above for the temporal component of the self four-force (34) up to a multiplicative factor of $-\gamma$, which arises because the force is defined by $f_{\mu}=d p_{\mu} / d \tau$, whereas the radiated power is equated to $d E / d t$. The minus sign results from the self force being the rate of change of the charge's energy, which is negative, whereas the radiated power is positive. We stress that for the case of the radiated power we used the far-field expressions for the electromagnetic field, namely, the field very far from the charge,

\begin{tabular}{|c|c|c|c|}
\hline Mode & Order & Contribution & Value of $m$ \\
\hline \multirow{3}{*}{$E_{1}$} & 1 & 1 & 1 \\
\hline & $\bar{\omega}^{2} r_{0}^{2}$ & $-\frac{2}{5}$ & 1 \\
\hline & $\bar{\omega}^{4} r_{0}^{4}$ & $\frac{43}{700}$ & 1 \\
\hline \multirow{4}{*}{$M_{1}$} & 1 & 0 & \\
\hline & $\bar{\omega}^{2} r_{0}^{2}$ & 0 & \\
\hline & $\bar{\omega}^{4} r_{0}^{4}$ & 0 & \\
\hline & 1 & 0 & \\
\hline \multirow[t]{2}{*}{$E_{2}$} & $\bar{\omega}^{2} r_{0}^{2}$ & $\frac{12}{5}$ & 2 \\
\hline & $\bar{\omega}^{4} r_{0}^{4}$ & $-\frac{16}{7}$ & 2 \\
\hline \multirow{4}{*}{$M_{2}$} & 1 & 0 & \\
\hline & $\bar{\omega}^{2} r_{0}^{2}$ & 0 & \\
\hline & $\bar{\omega}^{4} r_{0}^{4}$ & $\frac{1}{60}$ & 1 \\
\hline & 1 & 0 & \\
\hline \multirow{3}{*}{$E_{3}$} & $\bar{\omega}^{2} r_{0}^{2}$ & 0 & \\
\hline & $\bar{\omega}^{4} r_{0}^{0}$ & $\frac{729}{140}$ & 3 \\
\hline & & $\frac{1}{2100}$ & 1 \\
\hline \multirow{3}{*}{$M_{3}$} & 1 & 0 & \\
\hline & $\bar{\omega}^{2} r_{0}^{2}$ & 0 & \\
\hline & $\bar{\omega}^{4} r_{0}^{4}$ & 0 & \\
\hline
\end{tabular}
whereas for the case of the four force we calculated the near field, namely, the interaction of the field which the charge itself creates with the very same charge. In both cases we used only retarded fields.
Table I. The contribution to the radiated power of the first few multipole moments. First column: multipole moment in question. The capital letter is $E, M$ according to whether the mode is electric or magnetic. The subscript is the corresponding $l$. Second column: the order of the contribution. Third column: the contribution at that order. Fourth column: the value of $m$ wherefrom this contribution comes. Note that there is a factor of $\frac{2}{3} q^{2} \bar{\omega}^{4} r_{0}^{2}$ common to all modes which is suppressed in this table, and that the $E_{3}$ mode has contributions of the same order from two different values of $m$.

\section{POWER-SERIES EXPANSION OF THE RADIATION FIELD}

In order to gain more insight into the expression we derived for the radiated power for each $l m$ multipole moment, and to facilitate the summation over all modes, let us expand our expression in a power series. For each multipole mode we find a series expansion in $\bar{\omega}^{2} r_{0}^{2}$ such that only even powers of $\bar{\omega} r_{0}$ appear, as should be expected. This expansion is most easily obtained by using the standard expansions of the spherical Bessel functions for small values of their arguments. Next, we sum over all modes $m$ for a given value of $l$. The power series expansion can be obtained for all values of $l$. For example, for the dipole electric mode $(l=1)$ we find for the radiated power

$$
\mathcal{P}_{1}^{E}=\frac{2}{3} q^{2} \bar{\omega}^{4} r_{0}^{2}\left[1-\frac{2}{5} \bar{\omega}^{2} r_{0}^{2}+\frac{43}{700} \bar{\omega}^{4} r_{0}^{4}+O\left(\bar{\omega}^{6} r_{0}^{6}\right)\right] .
$$

This expression reflects the expansion of the radiated power in powers of the velocity squared. Our expressions for the radiated power in each $l m$ mode of the field [Eqs. (42) and (46)] are not restricted to slow motion, and are, in fact, fully relativistic. We find it convenient to expand in powers of the velocity (and thus obtain an expansion where the smallness parameter is the velocity) because this provides us with a very effective method to sum over all modes, which is translated into summation over all orders of the power expansion. When we sum over all orders in the expansion we are no longer restricted to the slow motion assumption, and we recover the fully relativistic nature of our analysis.

Table I summarizes our results for the first three multipole moments for both electric and magnetic radiative modes, up to contribution of order $\bar{\omega}^{8} r_{0}^{6}$. (All higher modes contribute 
only at higher orders in the power series expansion.) The first column of the table relates to the radiation mode, where $E_{l}$ means the $l$ multipole moment of electric radiation and correspondingly $M_{l}$ for magnetic modes. The second column describes the order of the contribution. (All modes have a common factor of $\frac{2}{3} q^{2} \bar{\omega}^{4} r_{0}^{2}$, which we suppress in this table.) The third column describes the contribution of the moment in question, and the fourth column says from what value of the magnetic number $m$ the contribution comes. Recall that we, in fact, sum over the contributions of $m$ and $-m$ modes, such that the contribution of the $m$ mode is just half of what Table I shows.

We find that the dipole magnetic modes do not radiate, and that the leading magnetic contribution comes from quadrupole radiation. This can be understood from the symmetry of the problem. Dipole magnetic radiation would correspond to a continuous current in a circular loop, say, with timedependent current, which creates a magnetic moment, the second time derivative of which does not vanish. In our case the current results from the uniform motion of a discrete pointlike charge, for which the second time derivative of the magnetic moment is zero. Therefore, the lowest moment for magnetic modes is quadrupole and not dipole as is the case for the electric modes. Because of the vanishing of the dipole magnetic modes, we find that the magnetic contribution is smaller than the electric contribution to the radiated power by a factor $\bar{\omega}^{4} r_{0}^{4}$. Whereas the dipole magnetic contribution to the radiated power vanishes for all orders in $\bar{\omega}^{2} r_{0}^{2}$, higher multipole magnetic modes do not. In general, dipole modes should have a contribution of order $\bar{\omega}^{4} r_{0}^{2}$, and for higherorder multipoles the leading contribution is smaller by a factor of $\bar{\omega}^{2(l-1)} r_{0}^{2(l-1)}$. This is the case for both electric and magnetic modes, but because of symmetry the dipole magnetic mode does not contribute. Consequently, the leading magnetic contribution comes from the quadrupole modes. Another point which should be made is the contribution of the different $m$ moments to a certain $l$. In the expressions we obtained for the radiated power, we see that electric modes have a multiplicative factor of $\bar{P}^{l m 2}(0)$, and magnetic modes have a factor of $\left[\partial \bar{P}^{l m}(0) / \partial \theta\right]^{2}$. Consequently, the contribution of electric modes vanishes whenever $l-m$ is odd, and the contribution of magnetic modes vanishes whenever $l-m$ is even. We are now in a position to see why the magnetic modes are so weak: The dipole mode does not contribute because with $l=1$, and bearing in mind that $m$ $=0$ modes do not radiate, the only remaining $m$ is $m=1$, but then $l-m=0$ is even, and the contribution of the dipole magnetic mode vanishes. Therefore, the leading magnetic contribution comes from the quadrupole mode. However, the $m=2$ modes do not contribute to the quadrupole magnetic mode, because in that case $l-m=0$ is even, and it is just the $m=1$ mode which can contribute. This results in the vanishing of the term of order $\bar{\omega}^{6} r_{0}^{4}$, and the leading magnetic contribution is only of order $\bar{\omega}^{8} r_{0}^{6}$.

\section{THE TOTAL RADIATED POWER}

We next sum all the contributions of the different multipole moments (the first few appear in Table I). The summation is done in the following way. All the terms in Table I have a common factor of $\frac{2}{3} q^{2} \bar{\omega}^{4} r_{0}^{2}$. The orders we shall refer to below are to powers of $\bar{\omega}^{2} r_{0}^{2}$ beyond this common term. At order 1 only the electric dipole mode contributes, with coefficient 1 . At order $\bar{\omega}^{2} r_{0}^{2}$ we have contributions from the electric dipole mode (with coefficient $-\frac{2}{5}$ ) and from the electric quadrupole mode (with coefficient $\frac{12}{5}$ ). The sum of these contributions gives a total coefficient 2. At order $\bar{\omega}^{4} r_{0}^{4}$ we have contributions from the electric dipole mode (with coefficient $\frac{43}{700}$ ), the electric quadrupole mode (with coefficient $-\frac{16}{7}$ ), the electric octupole mode (two terms, one with coefficient $\frac{729}{140}$, and the other with coefficient $\frac{1}{2100}$ ), and the magnetic quadrupole mode (with coefficient $\frac{1}{60}$ ). The sum of all these contributions gives a total coefficient of 3 . The coefficients of higher-order terms can be found analogously. Collecting all the contributions up to order $\bar{\omega}^{4} r_{0}^{4}$, we obtain for the radiated power

$$
\mathcal{P}=\frac{2}{3} q^{2} \bar{\omega}^{4} r_{0}^{2}\left[1+2 \bar{\omega}^{2} r_{0}^{2}+3 \bar{\omega}^{4} r_{0}^{4}+O\left(\bar{\omega}^{6} r_{0}^{6}\right)\right] .
$$

It can be shown (after a tedious calculation, or checking numerically) that for the $n$th order in the expansion inside the square brackets one finds the contribution to be $n \bar{\omega}^{2 n-2} r_{0}^{2 n-2}$, such that the general expression is

$$
\mathcal{P}=\frac{2}{3} q^{2} \bar{\omega}^{4} r_{0}^{2} \sum_{n=1}^{\infty} n \bar{\omega}^{2 n-2} r_{0}^{2 n-2} .
$$

This expression equals

$$
\mathcal{P}=\frac{2}{3} q^{2} \bar{\omega}^{4} r_{0}^{2} \frac{1}{\left(1-\bar{\omega}^{2} r_{0}^{2}\right)^{2}}=\frac{2}{3} q^{2} \gamma^{4} \bar{\omega}^{4} r_{0}^{2},
$$

which is the well-known expression for the radiated power of synchrotron radiation.

We showed above that the $I m$ mode of the radiated power corresponds to the $\mathrm{lm}$ mode of the temporal component of the self four-force. In fact, the only difference is an extra factor of $-\gamma$, common to all multipole moments. Therefore, summing all the components of the self force we find

$$
\hat{f^{t}}=-\frac{2}{3} q^{2} \gamma^{5} \bar{\omega}^{4} r_{0}^{2} .
$$

However, the self force

$$
f^{\hat{t}}=\frac{d p^{\hat{t}}}{d \tau}=\gamma \frac{d p^{\hat{t}}}{d t}=\gamma \frac{d E}{d t}=-\gamma \mathcal{P},
$$

as we indeed find. Here, $p_{\mu}$ is the four-momentum.

\section{COMPARISON WITH THE ABRAHAM-LORENTZ-DIRAC EQUATION}

We have obtained $f^{\hat{t}}$ by calculating the Lorentz force acting on the charge, where the force originates from the electromagnetic field which the very same charge creates. On the other hand, the self force on the charge is given by the Abraham-Lorentz-Dirac (ALD) equation, ${ }^{24,4}$ according to which

$$
f_{\mathrm{ALD}}^{\mu}=\frac{2}{3} q^{2}\left(\frac{D^{2} u^{\mu}}{d \tau^{2}}-u^{\mu} \frac{D u_{\alpha}}{d \tau} \frac{D u^{\alpha}}{d \tau}\right),
$$

where the operator $D$ denotes covariant differentiation. (Recall that even though space-time is flat, the coordinates are curved.) We shall next show that our result coincides with the ALD equation. Because the four-velocity is a constant four-vector, its partial derivative with respect to proper time 
vanishes. Therefore, the four-acceleration is given by

$$
a^{\mu}=\frac{D u^{\mu}}{d \tau}=\frac{D u^{\mu}}{d x^{\alpha}} u^{\alpha}=\Gamma_{\alpha \beta}^{\mu} u^{\alpha} u^{\beta},
$$

$\Gamma_{\alpha \beta}^{\mu}$ being the Christoffel symbols of the second kind. It can be readily verified that only the radial component of the fouracceleration does not vanish. Recall that only $u^{t}$ and $u^{\phi}$ are not zero. In spherical coordinates, the only nonzero component of the Christoffel symbols with lower indices $t$ or $\phi$ is $\Gamma_{\phi \phi}^{r}=-r \sin ^{2} \theta$. (Note that $\Gamma_{\phi \phi}^{\theta}=-\sin \theta \cos \theta$, but the cosine vanishes on the charge's world-line, and therefore this component does not contribute to the four-acceleration.) Bearing in mind that $\sin \theta=1$ on the world-line of the charge, we find that $a^{r}=-r_{0} \gamma^{2} \bar{\omega}^{2}$, such that the squared fouracceleration is $a_{\mu} a^{\mu}=r_{0}^{2} \gamma^{4} \bar{\omega}^{4}$. Substitution in the ALD equation (53) yields $f_{\mathrm{ALD}}^{\hat{t}}=-(2 / 3) q^{2} \gamma^{5} \bar{\omega}^{4} r_{0}^{2}$, namely, we find that

$$
f_{\mathrm{ALD}}^{\hat{t}}=f^{\hat{t}}
$$

Consequently, not only does our method for calculating the self force (by means of finding the Lorentz force acting on the charge by the field the very same charge produces) agree with the known expression for the radiated power at infinity, it also agrees with the Abraham-Lorentz-Dirac equation (53).

\section{DISCUSSION}

We presented a calculation for the temporal component of the self force for an electric charge in circular orbit in Minkowski space-time. The calculation is based on decomposing the field which the charge creates into Fourierharmonic modes, and finding the self force mode by mode. We derive the Lorentz force, and evaluate it on the world line of the charge. In the case of the temporal component it is possible to sum over all modes analytically. It turns out that this sum is finite, and is equal to the power carried by the emitted radiation to infinity. We also find agreement with the Abraham-Lorentz-Dirac equation.

We note that even though it appears that the agreement between the self force we calculated and the radiated power at infinity is instantaneous, we in fact expect both quantities to agree in general only after averaging over a full cycle. We find in this case the agreement to be instantaneous because the problem is stationary. Consequently, it happens that the instantaneous value of the force is also the average over a cycle. In time-dependent problems we do not expect the agreement to be instantaneous. We still expect both quantities to agree over a full period, or after integration over all time if the motion is nonperiodic.

One might be surprised that the locally derived self force turned out to be finite without any need of regularization. In general, one would indeed expect the self force to diverge. However, it is reasonable to expect the self force to be the sum of a divergent piece and the finite, physical piece. The divergent piece is believed to be proportional to the acceleration. In our case, only the radial component of the acceleration is not zero. In particular, the temporal component of the acceleration vanishes, such that one can expect the divergent piece of the temporal component of the self force to vanish. (One should define the pointlike limit carefully then.) This left us only with the finite piece, which is the physically important one.

We briefly remark on the spatial components of the self four-force. From symmetry, it is expected that the $\theta$ component should vanish. This is indeed what we find after a detailed calculation, similar to the calculation of the $t$ component. The $\phi$ component is proportional to the $t$ component. Specifically,

$$
\begin{aligned}
f^{\hat{\phi}}=q F^{\hat{\phi} \hat{\mu}} u_{\hat{\mu}} & =q F^{\hat{\phi} \hat{t}} u_{\hat{t}} \\
& =-q F^{\hat{t} \hat{\phi}} u_{\hat{t}}=-\frac{1}{\bar{\omega} r_{0}} q F^{\hat{t} \hat{\phi}} u_{\hat{\phi}}=-\frac{1}{\bar{\omega} r_{0}} f^{\hat{t}}
\end{aligned}
$$

This component of the self force is the component which was calculated in Ref. 11. We note that one can ask the following question: What external force should be exerted on a charged particle, in order to keep it in uniform circular motion? In addition to the mechanical centripetal force, there is also another force, which balances the electromagnetic self force. The additional external force which should be applied is just the minus of the $\phi$ component of the self force we calculated here. (See also Ref. 11.)

We also calculated the radial component of the self force using a similar approach. In that case, however, we were unable to sum over all modes analytically. This summation can still be done numerically. We find that this sum diverges, although each mode is finite. The divergence can be removed, and the renormalized result agrees with the Abraham-Lorentz-Dirac result for the radial component, which is zero. We used two regularization methods for the radial component: First, we model the charge to be extended, and then we consider the limit of the size of the charge approaching zero. In a sense, this approach is in the spirit of the classical electron models of Abraham, Lorentz, Poincaré, and others. The simplest model for a spatially extended particle is a dumbbell model, where the axis of the dumbbell is radial. (We do not argue that this is a realistic model for a charge. One hopes, nevertheless, that the self force on a pointlike charge would be independent of the model used in the limit of vanishing extension. In addition, the self force on a general extended object may be obtained by summing on all pairs of particles, where each pair is, in fact, a dumbbell.) After a simple mass renormalization (the bare mass is proportional to the acceleration of the dumbbell's center, and inversely proportional to the separation distance between the two edges of the dumbbell ${ }^{25}$ ), one indeed obtains the expected result of zero radial force. A second renormalization method is based on the observation that the $l$ mode of the radial force on a pointlike charge approaches a constant as $l \rightarrow \infty$. When one subtracts this asymptotic constant from each mode, the sum over all modes converges to the correct result. This latter regularization procedure is a direct application of Ori's prescription, ${ }^{10}$ which is successful also for more complicated and interesting cases. ${ }^{28}$

\section{ACKNOWLEDGMENTS}

I have benefited from invaluable discussions with Amos Ori. I thank Kip Thorne for reading the manuscript and for useful comments. At Caltech this research was supported by NSF Grant No. AST-9731698 and NASA Grant No. NAG56840 . 


\section{APPENDIX A: VECTOR SPHERICAL HARMONICS}

The electromagnetic vector potential is a four-vector, and therefore it cannot be decomposed into the usual scalar spherical harmonics, but instead needs to be decomposed into vector harmonics. However, because the Minkowski space-time is stationary (the metric functions are not functions of time), and thanks to the fact that the metric is diagonal, one can split the four-vector potential into a scalar (the temporal component) and a three-vector (the spatial components). The latter can then be decomposed into the usual vector spherical harmonics, whereas the former decomposes into the usual scalar spherical harmonics. Vector spherical harmonics are dealt with extensively in Ref. 19. Here we describe just their properties which are the most relevant ones for our needs. We shall use the notation and convention of Ref. 19. We shall also give the relation of the vector spherical harmonic we use to those given by Ref. 15 .

In order to decompose a general three-vector in flat spacetime, let us project it onto a tangent space to a sphere. Because this tangent space is two-dimensional, we need two base vectors in order to span it. These base vectors are most conveniently chosen to be the gradient of the usual scalar harmonics, and its dual, which in two dimensions is also a vector. $^{26}$ These are the Regge-Wheeler harmonics, ${ }^{27}$ defined by

$$
\psi_{a} \equiv a_{l} \frac{\partial}{\partial x^{a}} Y^{l m}
$$

and

$$
\phi_{a} \equiv b_{l} \epsilon_{a}^{b} \frac{\partial}{\partial x^{b}} Y^{l m}
$$

where $a_{l}$ and $b_{l}$ are some normalization factors, which we find explicitly below. The tensor $\epsilon_{a}^{b}$ can be represented by the matrix

$$
\epsilon_{a}^{b}=\left(\begin{array}{cc}
0 & -1 / \sin \theta \\
\sin \theta & 0
\end{array}\right),
$$

where the coordinates $x^{a}$ and $x^{b}$ can take the values $\theta$ or $\phi$. The constants $a_{l}$ and $b_{l}$ can be found as follows. We take the Regge-Wheeler harmonics $\psi_{a}$ and $\phi_{a}$, and integrate their scalar product over the sphere. One finds

$$
\int \psi_{a}^{l m *} \psi_{b}^{\tilde{l} \tilde{m}} g^{a b} d \Omega=a_{l}^{*} a_{l} l(l+1) \delta_{l i} \delta_{m \tilde{m}}
$$

and

$$
\int \phi_{a}^{l m *} \phi_{b}^{\tilde{l} \tilde{m}} g^{a b} d \Omega=b_{l}^{*} b_{l} l(l+1) \delta_{l l} \delta_{m \tilde{m}},
$$

and normalization yields $a_{l}=b_{l}=1 / \sqrt{l(l+1)}$. The metric $g_{a b}$ is the metric on the two-sphere, namely $d \Sigma^{2}$ $=g_{a b} d x^{a} d x^{b}=r^{2}\left(d \theta^{2}+\sin ^{2} \theta d \phi^{2}\right)$. The cross product of $\psi_{a}$ and $\phi_{a}$ vanishes, namely

$$
\int \psi_{a}^{l m} * \phi_{b}^{\tilde{l} \tilde{m}} g^{a b} d \Omega=0 .
$$

Next, because the metric is diagonal, we can explicitly list the components of the Regge-Wheeler harmonics in a normalized basis [namely, in a basis defined by $V_{\hat{\mu}}$ $=\sqrt{\left|g^{\mu \mu}\right|} V_{\mu}$ (no summation over repeated indices), where $V_{\mu}$ is any four-vector written in a covariant basis]:

$$
\begin{aligned}
& \psi_{\hat{\theta}}=\frac{1}{\sqrt{l(l+1)}} \frac{\partial Y^{l m}}{\partial \theta}, \\
& \psi_{\hat{\phi}}=\frac{1}{\sqrt{l(l+1)}} \frac{i m}{\sin \theta} Y^{l m}, \\
& \phi_{\hat{\theta}}=-\frac{1}{\sqrt{l(l+1)}} \frac{i m}{\sin \theta} Y^{l m}, \\
& \phi_{\hat{\phi}}=\frac{1}{\sqrt{l(l+1)}} \frac{\partial Y^{l m}}{\partial \theta} .
\end{aligned}
$$

We next denote $\psi_{\hat{a}}$ and $\phi_{\hat{a}}$ as $Y_{\hat{a}}^{E l m}$ and $Y_{\hat{a}}^{B l m}$, respectively, where $x^{a}$ can take the values $\theta$ or $\phi$. In addition to the tangent space, we also have the radial direction, which is orthogonal to the tangent space. However, the projection onto the radial direction would simply be just the scalar harmonics. Namely, we have $Y_{\hat{r}}^{R l m}=Y^{l m}$. Here $Y^{E}, Y^{B}$, and $Y^{R}$ are called collectively the pure-spin vector spherical harmonics.

Finally, we find it most convenient for our needs to define the pure-orbital vector harmonics, because they are eigenfunctions of the flat space-time Laplacian operator. These pure-orbital vector harmonics are built from the pure-spin harmonics in the following way:

$$
\begin{aligned}
& Y^{l+1, l m}=\frac{1}{\sqrt{2 l+1}}\left(\sqrt{l} Y^{E l m}-\sqrt{l+1} Y^{R l m}\right), \\
& Y^{l, l m}=-i Y^{B l m}, \\
& Y^{l-1, l m}=\frac{1}{\sqrt{2 l+1}}\left(\sqrt{l+1} Y^{E l m}+\sqrt{l} Y^{R l m}\right) .
\end{aligned}
$$

Namely, we find that $Y^{l, l m}$ is purely transverse, whereas the other two harmonics are two independent combinations of transverse and radial components. Table II gives the explicit expressions for the components of the pure-orbital vector spherical harmonics, in a normalized basis.

For completeness, we also give the relation between the pure-spin vector spherical harmonics and the vector spherical harmonics which are given, e.g., in Ref. 15 and involve the operator $\mathbf{L}=(1 / i) \mathbf{r} \times \nabla$. First, we define

$$
\mathbf{X}^{l m}=\frac{1}{\sqrt{l(l+1)}} \mathbf{L} Y^{l m} .
$$

[This definition is identical to Eq. (16.43) of Ref. 15.] Then,

$$
\begin{aligned}
& \mathbf{Y}^{B l m}=i \mathbf{X}^{l m}, \\
& \mathbf{Y}^{E l m}=-i \hat{\mathbf{r}} \times \mathbf{X}^{l m},
\end{aligned}
$$

and

$$
\mathbf{Y}^{R l m}=\hat{\mathbf{r}} Y^{l m}
$$

where $\hat{\mathbf{r}}$ is a unit vector in the radial direction.

It is useful to calculate the divergence of the pure-orbital vector harmonics. We find 
Table II. Pure-orbital vector harmonics in a normalized basis. The first column is the different components of the pure-orbital harmonics, and the second column is their value in terms of the scalar spherical harmonics.

\begin{tabular}{|c|c|}
\hline Component & Expression \\
\hline$Y_{\hat{r}}^{l+1, l m}$ & $-\sqrt{\frac{l+1}{2 l+1}} Y^{l m}$ \\
\hline$Y_{\hat{r}}^{l, l m}$ & 0 \\
\hline$Y_{\hat{r}}^{l-1, l m}$ & $\sqrt{\frac{l}{2 l+1}} Y^{l m}$ \\
\hline \multirow{2}{*}{$Y_{\hat{\theta}}^{l+1, l m}$} & $1 \quad \partial Y^{l m}$ \\
\hline & $\overline{\sqrt{(l+1)(2 l+1)}} \overline{\partial \theta}$ \\
\hline$Y_{\hat{\theta}}^{l, l m}$ & $-\frac{1}{\sqrt{l(l+1)}} \frac{m}{\sin \theta} Y^{l m}$ \\
\hline \multirow{2}{*}{$Y_{\hat{\theta}}^{l-1, l m}$} & $1 \quad \partial Y^{l m}$ \\
\hline & $\sqrt{l(2 l+1)} \overline{\partial \theta}$ \\
\hline \multirow[b]{2}{*}{$Y_{\hat{\phi}}^{l+1, l m}$} & $1 \quad \mathrm{im}$ \\
\hline & $\overline{\sqrt{(l+1)(2 l+1)}} \overline{\sin \theta} Y^{m}$ \\
\hline \multirow{2}{*}{$Y_{\hat{\phi}}^{l, l m}$} & $1 \quad \partial Y^{l m}$ \\
\hline & $-i \overline{\sqrt{l(l+1)}} \overline{\partial \theta}$ \\
\hline \multirow{2}{*}{$Y_{\hat{\phi}}^{l-1, l m}$} & $1 \quad \stackrel{i m}{=} Y^{l m}$ \\
\hline & $\sqrt{l(2 l+1)} \overline{\sin \theta} Y^{m}$ \\
\hline
\end{tabular}

$$
\begin{aligned}
\nabla \cdot \mathbf{Y}^{l^{\prime}, l m}= & -\left(l^{\prime}-l\right)^{2}\left[l^{\prime}+\frac{1}{2}\left(l^{\prime}-l+1\right)\right] \\
& \times \sqrt{\frac{l^{\prime}+\left(l^{\prime}-l\right)\left(l^{\prime}-l+1\right) / 2}{2 l+1}} \frac{1}{r} Y^{l m},
\end{aligned}
$$

where $l^{\prime}$ takes the values $l-1, l$, or $l+1$. The pure-spin harmonics satisfy

$$
\begin{aligned}
& \nabla \cdot \mathbf{Y}^{R l m}=\frac{2}{r} Y^{l m}, \\
& \nabla \cdot \mathbf{Y}^{B l m}=0, \\
& \nabla \cdot \mathbf{Y}^{E l m}=-\frac{\sqrt{l(l+1)}}{r} Y^{l m} .
\end{aligned}
$$

With these expressions for the divergence of the vector spherical harmonics, it can be readily shown that the Lorenz gauge condition is satisfied separately by each multipole moment of the field, by a direct substitution of the expressions we found for the components of the electromagnetic vector potential.

\section{APPENDIX B: SOLUTION OF A DIFFERENTIAL EQUATION WITH A DELTA FUNCTION SOURCE}

In this appendix we shall show how one can find the solution to the inhomogeneous second-order ordinary differential equation

$$
g(x) y^{\prime \prime}(x)+q(x) y^{\prime}(x)+h(x) y(x)=f(x) \delta\left(x-x_{0}\right),
$$

when the two independent solutions $u(x)$ and $v(x)$ of the corresponding homogeneous equation $g(x) y^{\prime \prime}(x)$ $+q(x) y^{\prime}(x)+h(x) y(x)=0$ are known. By a prime we denote in this appendix differentiation with respect to $x$.

We first assume that because the source has a delta function form, we shall have one functional form for the solution for $x>x_{0}$, and another form for $x<x_{0}$. Specifically, let us seek a solution of the form

$$
y(x)=A u(x) \Theta\left(x-x_{0}\right)+B v(x) \Theta\left(x_{0}-x\right) .
$$

Here, $\Theta(x)$ is the Heaviside step function, which satisfies $\Theta(x)=1$ for $x>0$ and $\Theta(x)=0$ for $x<0$. The problem, then, is to find the constant coefficients $A, B$, such that we shall find the unique solution for the given inhomogeneous equation. Let us denote $A(x) \equiv A \Theta\left(x-x_{0}\right)$ and $B(x)$ $\equiv B \Theta\left(x_{0}-x\right)$. Then, the solution is of the form $y(x)$ $=A(x) u(x)+B(x) v(x)$. Differentiating $A(x)$ and $B(x)$ we find that $A^{\prime}(x)=A \delta\left(x-x_{0}\right)$ and $B^{\prime}(x)=-B \delta\left(x_{0}-x\right)$. Consequently,

$$
\begin{aligned}
y^{\prime}(x)= & A(x) u^{\prime}(x)+A^{\prime}(x) u(x)+B(x) v^{\prime}(x) \\
& +B^{\prime}(x) v(x) .
\end{aligned}
$$

According to the variation of the parameters method, we now demand that $A^{\prime}(x) u(x)+B^{\prime}(x) v(x)=0$. Substituting the expression for $A^{\prime}(x)$ and $B^{\prime}(x)$ we demand that $A \delta(x$ $\left.-x_{0}\right) u(x)-B \delta\left(x_{0}-x\right) v(x)=0$, or $\left[A u\left(x_{0}\right)-B v\left(x_{0}\right)\right] \delta(x$ $\left.-x_{0}\right)=0$. Specifically, we find that $B=A u\left(x_{0}\right) / v\left(x_{0}\right)$.

Then, we find that

$$
\begin{aligned}
y^{\prime}(x) & =A(x) u^{\prime}(x)+B(x) v^{\prime}(x) \\
& =A u^{\prime}(x) \Theta\left(x-x_{0}\right)+B v^{\prime}(x) \Theta\left(x_{0}-x\right)
\end{aligned}
$$

and

$$
\begin{aligned}
y^{\prime \prime}(x)= & A u^{\prime}(x) \delta\left(x-x_{0}\right)+A u^{\prime \prime}(x) \Theta\left(x-x_{0}\right) \\
& -B v^{\prime}(x) \delta\left(x_{0}-x\right)+B v^{\prime \prime}(x) \Theta\left(x_{0}-x\right) .
\end{aligned}
$$

We next substitute these expressions in the differential equation. We use now the fact that the functions $u(x)$ and $v(x)$ solve the homogeneous equation to eliminate all the terms in the equation which are proportional to the Heaviside step function. We are thus left with

$$
\begin{aligned}
& g(x) A u^{\prime}(x) \delta\left(x-x_{0}\right)-g(x) B v^{\prime}(x) \delta\left(x-x_{0}\right) \\
& -f(x) \delta\left(x-x_{0}\right)=0 .
\end{aligned}
$$

The solution for this is

$$
A u^{\prime}\left(x_{0}\right)-B v^{\prime}\left(x_{0}\right)=f\left(x_{0}\right) / g\left(x_{0}\right) .
$$

Recalling that $B=A u\left(x_{0}\right) / v\left(x_{0}\right)$ we readily find that

$$
A=\frac{f\left(x_{0}\right)}{g\left(x_{0}\right)} \frac{v\left(x_{0}\right)}{v\left(x_{0}\right) u^{\prime}\left(x_{0}\right)-u\left(x_{0}\right) v^{\prime}\left(x_{0}\right)}=\frac{f\left(x_{0}\right)}{g\left(x_{0}\right)} \frac{v\left(x_{0}\right)}{W\left(x_{0}\right)},
$$

where $W$ is the Wronskian of the two functions $v$ and $u$. We thus find that $A=\left[f\left(x_{0}\right) / g\left(x_{0}\right)\right] v\left(x_{0}\right) / \mathrm{W}\left(x_{0}\right)$, and $B$ $=\left[f\left(x_{0}\right) / g\left(x_{0}\right)\right] u\left(x_{0}\right) / \mathrm{W}\left(x_{0}\right)$, such that the solution for the differential equation is given by 


$$
\begin{aligned}
y(x)= & \frac{f\left(x_{0}\right)}{g\left(x_{0}\right)} \frac{v\left(x_{0}\right)}{W\left(x_{0}\right)} u(x) \Theta\left(x-x_{0}\right) \\
& +\frac{f\left(x_{0}\right)}{g\left(x_{0}\right)} \frac{u\left(x_{0}\right)}{W\left(x_{0}\right)} v(x) \Theta\left(x_{0}-x\right) .
\end{aligned}
$$

${ }^{\text {a)} E l e c t r o n i c ~ m a i l: ~ b u r k o @ t a p i r . c a l t e c h . e d u ~}$

T. Erber, "The classical theories of radiation reaction,' Fortschr. Phys. 9, 343-392 (1961)

${ }^{2}$ P. Pearle, "Classical electron models," in Electromagnetism-Paths to Research, edited by D. Teplitz (Plenum, New York, 1982).

${ }^{3}$ F. Rohrlich, Classical Charged Particles (Addison-Wesley, Reading, MA, 1965).

${ }^{4}$ P. A. M. Dirac, 'Classical theory of radiating electrons,' Proc. R. Soc. London, Ser. A 167, 148-169 (1938).

${ }^{5}$ B. S. DeWitt and R. W. Brehme, "Radiation damping in a gravitational field,'” Ann. Phys. (N.Y.) 9, 220-259 (1960).

${ }^{6}$ T. C. Quinn and R. M. Wald, "Axiomatic approach to electromagnetic and gravitational radiation reaction of particles in curved spacetime,", Phys. Rev. D 56, 3381-3394 (1997).

${ }^{7}$ J. D. Templin, "Radiation reaction and runaway solutions in acoustics,", Am. J. Phys. 67, 407-413 (1999).

${ }^{8}$ A. Ori, "Radiative evolution of orbits around a Kerr black hole,' Phys. Lett. A 202, 347-351 (1995).

${ }^{9}$ A. Ori, "Radiative evolution of the Carter constant for generic orbits around a Kerr black hole,', Phys. Rev. D 55, 3444-3456 (1997).

${ }^{10} \mathrm{~A}$. Ori (unpublished); L. Barack and A. Ori, "Mode sum regularization approach for the self force in black hole spacetime', (submitted to Phys. Rev. D), gr-qc/9912010.

${ }^{11}$ T. H. Boyer, "Mass renormalization and radiation damping for a charged particle in uniform circular motion,'” Am. J. Phys. 40, 1843-1846 (1972).

${ }^{12} \mathrm{~S}$. Turner (ed.), Synchrotron Radiation and Free Electron Lasers (CERN 98-04, 1996) (unpublished).

${ }^{13}$ G. Margaritondo, Introduction to Synchrotron Radiation (Oxford U.P., Oxford, 1988)

${ }^{14}$ A. A. Sokolov and I. M. Ternov, Radiation from Relativistic Electrons (American Institute of Physics, New York, 1986).

${ }^{15}$ J. D. Jackson, Classical Electrodynamics, 2nd ed. (Wiley, New York, 1975).
${ }^{16}$ L. D. Landau and E. M. Lifshitz, The Classical Theory of Fields, 4th ed. (Pergamon, Oxford, 1975).

${ }^{17}$ T. Erber, G. B. Baumgartner, Jr., D. White, and H. G. Latal, “Mega-Gauss Bremsstrahlung and radiation reaction," in Proceedings of the 12th International Conference on High-energy Accelerators, edited by F. T. Cole and R. Donaldson (Fermilab, 1983).

${ }^{18}$ B. F. Schutz, A First Course in General Relativity (Cambridge U.P., Cambridge, 1985).

${ }^{19}$ K. S. Thorne, "Multipole expansions of gravitational radiation,' Rev. Mod. Phys. 52, 299-339 (1980).

${ }^{20}$ The first author to write this gauge condition was L. V. Lorenz. I thank Asher Peres for drawing my attention to this. Cf. B. Felsager, Geometry, Particles, and Fields (Springer, New York, 1998).

${ }^{21}$ The Lorenz gauge is consistent with the multipole decomposition, in the sense that the Lorenz gauge is satisfied separately by each multipole mode (see Appendix A).

${ }^{22}$ The overall sign of the Wronskian determinant is determined unambiguously by the requirement that Eq. (6) would be the solution of the inhomogeneous equation (5). See Appendix B.

${ }^{23}$ G. B. Arfken, Mathematical Methods for Physicists, 3rd ed. (Academic, Orlando, 1985), p. 677. Note, however, a typographical misprint.

${ }^{24}$ Note that the form of the ALD equation depends on the choice of metric signature. Our metric signature here is +2 . For a -2 metric signature the minus sign turns into a plus sign. (This happens because the four-velocity normalization is $u_{\alpha} u^{\alpha}=-1$ if the signature is +2 , and $u_{\alpha} u^{\alpha}=1$ if the signature is -2 .) The correct relative sign between the two terms in the ALD equation is most easily found by checking that the four-force is perpendicular to the four-velocity.

${ }^{25}$ D. J. Griffiths and R. E. Owen, "Mass renormalization in classical electrodynamics,' Am. J. Phys. 51, 1120-1126 (1983).

${ }^{26}$ V. D. Sandberg, "Tensor spherical harmonics on $S^{2}$ and $S^{3}$ as eigenvalue problems,' J. Math. Phys. 19, 2441-2446 (1978).

${ }^{27}$ T. Regge and J. A. Wheeler, "Stability of a Schwarzschild singularity," Phys. Rev. 108, 1063-1069 (1957).

${ }^{28}$ L. M. Burko, "Self-force on static charges in Schwarzschild spacetime,", Class. Quantum Grav. 17, 227-250 (2000); L. M. Burko, "Self-force on particle in orbit around a black hole", (in preparation); L. M. Burko, "Self-force approach for radiation reaction," in Proceedings of the 3rd Amaldi Conference on Gravitational Waves, edited by Sydney Meshkov (to be published), gr-qc/9911089.

\section{LEBESGUE AND RIEMANN INTEGRATION}

Indeed, for more than 40 years I have claimed that if whether an airplane would fly or not depended on whether some function that arose in its design was Lebesgue but not Riemann integrable, then I would not fly in it. Would you? Does Nature recognize the difference? I doubt it!

R. W. Hamming, “Mathematics on a Distant Planet,' Am. Math. Monthly 105 (7), 640-650 (1998). 